\section{Consultório na Rua: visibilidades, invisibilidades e hipervisibilidade}

\author{
Street Outreach Offices: visibility, invisibility, and \\ enhanced visibility
}

\author{
La consulta médica en la calle: visibilidades, \\ invisibilidades e hipervisibilidades
}

\begin{abstract}
This article discusses care for street people from a socio-anthropological perspective, using participant observation conducted with a team from a street outreach project. Based on observations, street people are historically viewed as marginal and rarely obtain access to health services, thus making them invisible to the Brazilian Unified National Health System. Brazil's National Pol icy for the Homeless provides for their access to health care, but such care is not always guaranteed in practice, because health services and professionals have little experience in dealing with homeless persons. The study concludes that enhanced visibility is needed to ensure care for people living on the street, establishing a therapeutic bond that deconstructs stigmatizing practice.

Homeless Persons; Medical Sociology; Health Policy
\end{abstract}

Janaína Alves da Silveira Hallais 1 Nelson Filice de Barros 1

\section{Resumo}

Este artigo reflete sobre o acolhimento e a produção de cuidado destinados à população em situação de rua numa perspectiva socioantropológica, a partir de uma observação participante realizada junto a uma equipe de Consultório na Rua. Observou-se que essa população, historicamente visibilizada como marginal, dificilmente consegue acessar os serviços de saúde, tornandose invisível ao próprio Sistema Único de Saúde. Embora a Política Nacional para a População em Situação de Rua pretenda assegurar acesso à saúde, o cuidado, no entanto, nem sempre é garantido porque serviços e profissionais de saúde têm pouca experiência para acolher pessoas em situação de rua e atender as suas necessidades. Conclui-se que o desafio é hipervisibilizar as linhas de cuidado para situações impostas pela vida na rua e a construção de vínculo terapêutico desconstrutor da prática estigmatizante.

Moradores de Rua; Sociologia Médica; Política de Saúde 


\section{Introdução}

A Política Nacional para a População em Situação de Rua (PNPSR), instituída pelo Decreto Presidencial no 7.053 1, de 23 de dezembro de 2009, caracteriza como "população em situação de rua": "o grupo populacional heterogêneo que possui em comum a pobreza extrema, os vínculos familiares interrompidos ou fragilizados e a inexistência de moradia convencional regular, e que utiliza os logradouros públicos e as áreas degradadas como espaço de moradia e de sustento, de forma temporária ou permanente, bem como as unidades de acolhimento para pernoite temporário ou como moradia provisória" (Artigo 1, parágrafo único).

Embora a PNPSR tenha como um de seus objetivos assegurar cuidados de saúde, os serviços assistenciais e de albergamento não dispõem de estrutura e recursos humanos para o atendimento à saúde e não apresentam relações bem estruturadas com a rede de atenção básica. Nesse contexto, um dos desafios para a gestão municipal é implantar uma política intersetorial que contemple ações entre as Secretarias de Assistência, Saúde, Habitação, e Trabalho e Renda, por exemplo. A ausência dessa rede dificulta o cumprimento da equidade no atendimento da população em situação de rua nas unidades básicas de saúde, que geralmente conseguem atenção em serviços de urgência/emergência.

Para sanar tais problemas e melhorar a capacidade de resposta às demandas e necessidades de saúde inerentes à população em situação de rua, o Ministério da Saúde publicou a Portaria no 122 2, de 25 de janeiro de 2012, que define as diretrizes de organização e funcionamento das Equipes de Consultório na Rua como porta de entrada deste segmento populacional nos serviços de atenção. Assim, o Consultório na Rua (CnaR) é um equipamento itinerante de saúde que integra a Rede de Atenção Básica e desenvolve ações de atenção psicossocial, devendo seguir os fundamentos e as diretrizes estabelecidas pela Política Nacional de Atenção Básica. Por isso, trabalha em parceria com as unidades básicas de saúde e com os Centros de Atenção Psicossocial, priorizando que a assistência e o cuidado sejam realizados no próprio território de rua 3 .

A assistência em saúde oferecida pelo CnaR está direcionada para acolher demandas diversificadas e complexas, considerando a "saúde não centrada somente na assistência aos doentes, mas, sobretudo, na promoção de saúde e no resgate da qualidade de vida, com intervenção nos fatores que a colocam em risco" 4 (p. 34), abrangendo também atendimento para problemas ou complicações advindas do abuso de álcool e outras drogas.
No entanto, a produção de cuidado destinada à população em situação de rua é quase inexiste, não apenas por se tratar de uma política pública muito recente 5 , mas também pela sua escassez na literatura científica 5,6, configurando uma lacuna de conhecimento sobre esta problemática. Diante do contexto apresentado, foi observada a importância de refletir sobre o desafio de instituir linhas de cuidado que atendam as necessidades impostas pela vida na rua e que viabilizem a criação de vínculo entre os profissionais de saúde e os indivíduos em situação de rua, identificando as visibilidades, invisibilidades e hipervisibilidades que marcam o trabalho em saúde com essa população. O objetivo deste artigo é, portanto, refletir acerca do cuidado destinado a PSR numa perspectiva socioantropológica, a partir de observação participante realizada junto a uma equipe de CnaR.

\section{Método}

Por meio da observação participante foi possível acompanhar uma equipe CnaR em campo, em reuniões internas, intersetoriais e no acolhimento e cuidado de pacientes em consultas e internações em diferentes serviços de saúde, durante o período de maio a dezembro de 2013. Tal experiência conferiu um duplo desafio: atuar como cientista social junto a profissionais de saúde 7 e (re)conhecer a dinâmica do cuidado na rua. O trabalho de campo e a produção dos dados empíricos foram organizados com base em anotações em um diário de campo, no qual foram registrados acontecimentos, relatos de interlocutores, descrições de algumas atividades, dentre outras reflexões e percepções da pesquisadora, que foram posteriormente organizados e analisados de forma temática.

A equipe acompanhada teve suas atividades iniciadas em outubro de 2012 e dispõe de 13 profissionais: um psicólogo, um enfermeiro, dois técnicos de enfermagem, um assistente social, um terapeuta ocupacional, dois médicos, três redutores de danos, um motorista e um coordenador. O deslocamento da equipe é feito em uma unidade móvel, onde também os pacientes são transportados quando necessário. Para maior efetividade das ações em saúde, o CnaR desenvolve projetos intersetoriais com outros serviços e instituições das áreas da saúde, da assistência e do judiciário, de modo a oferecer melhores respostas à população em situação de rua. Suas parcerias no campo da saúde são, principalmente, com unidades de saúde e hospitais públicos da cidade, com o objetivo de realizar consultas, exames laboratoriais e outros procedimentos. 
Em muitas situações participei da distribuição de copos d'água, preservativos e protetores labiais; da organização das pessoas que queriam conversar com os profissionais de saúde; em campanhas de vacinação e em conversas com sujeitos que procuravam a equipe. $\mathrm{O}$ envolvimento nessas atividades e o uso da camiseta amarela do CnaR, símbolo da equipe, os "amarelinhos", proporcionaram a aproximação dos usuários do serviço, que queriam entender o meu papel ali dentro, como cientista social. A condição "liminar”, de estranhamento, não impossibilitou várias trocas entre nossas identidades, subjetividades e narrativas, sobre o fato de viver na rua, de como sobreviver e suas reivindicações.

O recurso da observação participante foi justificado pela capacidade interacional que esta metodologia propicia ao pesquisador, aproximando-o dos agentes sociais, de suas práticas e vivências cotidianas. Esse método é bastante peculiar à Antropologia, reconhecido no século XX como uma legítima ferramenta de pesquisa no âmbito acadêmico-científico pelo antropólogo polonês Bronislaw Malinowski ${ }^{8}$. Na contemporaneidade, destaca-se o debate sobre os limites éticos e legais desse tipo de pesquisa. A antropóloga Linda Smith Tuhiwai 9 apresenta uma reflexão crítica sobre o discurso ocidental sobre o "outro", que tende a operar num sentido colonizador, reduzindo os sujeitos da pesquisa a meros objetos. Orientada por metodologias descolonizadoras, Tuhiwai discorre sobre a importância de desconstruir o olhar imperialista que orienta muitos pesquisadores em campo.

Com base nesses apontamentos, este trabalho foi direcionado para a pluralidade e polifonia dos agentes e instituições que interagem no contexto da rua, priorizando suas narrativas e descentralizando os discursos institucionalizados, atribuindo novos significados a partir das experiências dos próprios interlocutores 10 , para “questionar as formas de representação, produção de conhecimento, experiência, método e teoria sobre o outro" 11 (p. 586).

"Olhar de perto e de dentro" permitiu ainda inquietações e questionamentos sobre a criação de vínculo, a produção de cuidado e as possíveis formas de interação entre profissionais da saúde e pacientes 12. Para tanto, ao longo do processo foi preciso também desenvolver leituras dirigidas sobre temáticas como cuidado 13,14 , população de rua 1,4,5,6, atenção primária em saúde 15 , descolonização 9,16,17, interculturalidade 18,19 , políticas públicas de saúde 3,20 e diversidade cultural 16 . Tais leituras forneceram subsídios para direcionar e apurar o olhar em campo e apreender as relações desenvolvidas entre os profissionais do CnaR e os usuários do serviço.

\section{Resultados e discussão}

Ainda que haja a PNPSR e outras políticas setoriais e intersetoriais, elas nem sempre garantem um cuidado integral pois o imaginário social sobre a população em situação de rua influencia significativamente a prática dos profissionais que atuam nos serviços de saúde. Assim, o princípio de integralidade do Sistema Único de Saúde (SUS) e outras diretrizes da Política Nacional de Humanização 21 deixam de ser cumpridos quando, por exemplo, o CnaR não consegue internar pessoas em condição de rua em hospitais públicos pelo fato de as equipes hospitalares considerarem que a alta é complicada por não possuírem endereço fixo e rede de apoio para recuperação. Características estigmatizantes como a sujeira, o mau cheiro e o efeito de drogas lícitas e ilícitas são, muitas vezes, determinantes para a precariedade no acolhimento ao morador de rua nos serviços de saúde. O processo de exclusão dessa população é reforçado institucionalmente por meio da burocracia para o agendamento das consultas e da inflexibilização dos horários de atendimento, assim como da exigência de documento de identidade, comprovante de endereço e do Cartão SUS.

\section{Visibilidades}

De acordo com a Secretaria Municipal de Cidadania, Assistência e Inclusão Social do Município de Campinas, São Paulo, Brasil, foram registradas na cidade, em 2013, 601 pessoas em situação de rua 22. A atenção assistencial a essa população é executada pelo Departamento de Operações de Assistência Social, por intermédio da Coordenadoria Setorial de Proteção Social Especial de Alta Complexidade - População Adulta em Situação de Rua, que disponibiliza serviços municipais de albergamento por meio do Serviço de Atendimento ao Migrante, Itinerante e Mendicante (SAMIM), Abrigo Municipal Renascer, Serviço de Acolhimento e Referenciamento Social (SARES) e do Centro Popular de Serviço de Acolhimento e Referenciamento Social (Centro Pop) 22. Entretanto, tais programas são insuficientes para sanar as necessidades da população de rua, funcionando apenas eventualmente como abrigo noturno 23 .

Inicialmente, as ações do CnaR eram desenvolvidas apenas na região central da cidade, que foi divida em sete campos de atuação: Catedral, Praça Rui Barbosa, Terminal Central, Mercado Municipal, Praça do Carmo, SAMIM e Praça do Fórum. Seguindo a dinâmica que o próprio campo impõe, foi necessário expandir a área de atuação e incluir outras áreas da cidade, devido à migração causada por uma ação da Secretaria 
de Segurança Pública, que afastou um expressivo número de moradores de rua de seus territórios.

No segundo semestre de 2013, foi iniciada a operação "Centro Seguro”, implementada pela Secretaria Municipal de Cooperação nos Assuntos de Segurança Pública, que intensificou o patrulhamento na região central, principalmente nos pontos considerados mais críticos por causa da aglomeração de pessoas em situação de rua. O objetivo foi coibir furtos, tráfico e consumo de drogas como garantia de proporcionar mais segurança aos moradores, lojistas e frequentadores da região. As intervenções realizadas pela Guarda Municipal tinham como finalidade apreender drogas, armas usadas para cometer delitos e, também, cadastrar e encaminhar as pessoas abordadas para serviços assistenciais e de saúde mental. Concomitantemente a essa operação, uma série de reformas em prédios públicos também serviram para dispersar os grupos que ficavam no entorno, principalmente com a demolição de um serviço de albergamento que ficava nas imediações de um dos terminais de ônibus do centro da cidade, por exemplo.

A operação "Cata-treco", do Departamento de Limpeza Urbana do Município é outra ação do poder público que penaliza a população em situação de rua. Com a retaguarda da Guarda Municipal, um caminhão percorre periodicamente as ruas do centro da cidade coletando materiais considerados entulho, muitas vezes, pertences como: bolsas, mochilas, malas, livros, colchões, papelões, cobertores, calçados e roupas, gerando tumulto em muitas ocasiões, principalmente quando são recolhidos documentos de identidade, carteira de trabalho, medicamentos, fotos e outros registros pessoais.

Essas ações de regulação e controle 24 não visam ao cuidado das pessoas em situação de rua, mas a revitalização de espaços urbanos considerados degradados. Essas dinâmicas institucionais se configuram como práticas de biopoder 24 , nas quais a "visibilidade" não tem sentido de reconhecimento social, legitimando e reforçando ainda mais o distanciamento e a desigualdade socioestrutural 23 .

A transformação do espaço da rua em moradia nos leva a refletir sobre os "efeitos de lugar" 25 , como a visibilidade imediata que se tem, uma vez que tem significado social e um potencial de exclusão. A cristalização e a naturalização do estigma reforçam substancialmente o distanciamento e as diferenciações entre os grupos, recaindo diretamente em classificações que acabam por produzir rotulações distorcidas da realidade, facilmente compartilhadas e aceitas sem cautela ou sem debate. Frequentemente a grande mídia tem classificado e apresentado os "moradores de rua" como um grande problema urbano. Entre os discursos midiáticos, sobressai aquele que associa esse grupo social com a chamada "cracolândia”, que é apontada como um espaço degradante das médias e grandes cidades brasileiras 26,27 . Esse ideário, dominante e estigmatizante, deixa invisível a condição de vulnerabilidade física e social que assola a população em situação de rua, projetando uma visibilidade negativa sobre essas pessoas e interferindo no reconhecimento desses indivíduos como sujeitos sociais de direitos constitucionais. Essa perspectiva faz com que as pessoas em situação de rua, ao invés de serem consideradas como grupo em maior vulnerabilidade, sejam identificadas como causadoras de insegurança e violência urbana 23 . Tais condições contribuem para que esses próprios indivíduos sintam-se excluídos pela sociedade 6 .

\section{Invisibilidades}

Ao mesmo tempo em que as ações do poder público deixam visível a violência estrutural sobre a população em situação de rua, também conferem "invisibilidades" ao negarem o pertencimento deste grupo social na sociedade 20 , sugerindo que são "incômodos" que devem ser invisibilizados e silenciados em seus direitos e reivindicações.

A condição de invisibilidade é reforçada, ainda, pela falta de documentação necessária para acessar serviços e benefícios sociais que o Estado garante. A “indocumentação" 14, um problema muito comum entre os indivíduos que estão em situação de rua, impede, por exemplo, o cadastro em unidades de atenção básica ou a retirada de remédios de uso controlado, já que é preciso apresentar documento de identidade e comprovante de endereço para a confecção do Cartão SUS, embora o Artigo 19 da Portaria $n^{\circ} 940$ 28, que regulamenta o Sistema Cartão Nacional de Saúde, dispense a comprovação de endereço domiciliar da população em situação de rua.

Além do poder público investir pouco em ações de inclusão, de reconhecimento da população em situação de rua e em políticas públicas que promovam, efetivamente, a equidade desses sujeitos no acesso a oportunidades, observa-se, segundo Varanda \& Adorno 29, que isto indica a incapacidade do poder público em lidar com as demandas da população em situação de rua, além de reforçar a punição ao indivíduo pela sua própria situação.

Se, por um lado, o discurso científico e acadêmico tem defendido que a problemática da população em situação de rua é uma questão de saúde pública e de políticas sociais 23,29,30, por outro, ainda há o equívoco de apresentar causali- 
dade entre viver nas ruas com o consumo de drogas e a violência, numa perspectiva pouco crítica, que acaba por reiterar um discurso que perpassa pela ordem do controle.

Em pesquisa realizada com uma equipe de CnaR na cidade de Recife, Pernambuco, Silva et al. 6 tomaram como pressuposto que existem dois fenômenos distintos que se interligam: o viver nas ruas e o uso de drogas. Após a realização do trabalho, também qualitativo e com a técnica de observação participante, concluíram que as práticas da equipe privilegiaram o vínculo e o acolhimento das populações em situação de rua, reforçando a importância do protagonismo, da interdisciplinaridade e do trabalho em rede intersetorial. Embora esse trabalho contribua para preencher a lacuna de conhecimento existente e de defender a importância dos profissionais de saúde acessarem os usuários de drogas em situação de rua, desenvolve uma relação direta entre viver nas ruas e a mendicância e o consumo de drogas, sem avançar numa discussão mais apurada sobre a condição de invisibilidade que atinge a população em situação de rua. Toma um problema multifatorial e orienta suas práticas pela perspectiva mais cara ao campo da saúde de intervir e prescrever a vida dos cidadãos. Além disso, o artigo não menciona que a partir das Portarias no 122 e no 123, ambas de 25 de janeiro de 2012, as equipes de "Consultório de Rua", inicialmente ligadas à Coordenação Nacional de Saúde Mental, passaram a se chamar "Consultório na Rua”, integrando os serviços da atenção básica 31 .

Aprofundar essa questão é importante, pois o uso e os efeitos de substâncias psicoativas não são o único fator de risco que afeta a população em situação de rua ou que fragiliza o seu estado de saúde. Apesar de existirem políticas de promoção da equidade e do Ministério da Saúde coordenar ações e programas orientados para atender a população em situação de rua ${ }^{32}$, estas ofertas ainda têm se mostrado insuficientes para suprirem as necessidades deste grupo, sobretudo os problemas de saúde que os afetam em decorrência da precariedade e falta de cuidado. Em geral, os problemas de saúde mais comuns e urgentes apresentados pela população observada são tuberculose, DSTs, hepatites virais, doenças dermatológicas, além de transtornos mentais e variados tipos de sofrimento psíquico 4 . A dificuldade no cumprimento dos direitos e as diversas formas de vulnerabilidades que atingem essa população, como exposição às variações climáticas, condições insalubres de moradia e de alimentação, e ações de repressão por parte de políticas de segurança pública, afetam negativamente a condição de saúde dos indivíduos em situação de rua.
As condições de precariedade, privação e invisibilidade de direitos em que vivem essas pessoas exigem que se busquem alternativas para os problemas, pois a oferta de cuidados do SUS tem sido limitada. A proeminente dificuldade no acesso aos serviços é um reflexo do processo de exclusão e "invisibilização" que marca a população em situação de rua. Como destacado por Starfield 15, existe uma relação entre disparidades sociais e desigualdade nos acessos aos serviços de saúde, que obedecem a um fator estrutural em que disparidades socioeconômicas estão associadas com condições desiguais nos acessos aos serviços e na condição de saúde das populações. O desafio, portanto, não está somente no cumprimento da universalidade e da equidade, mas na qualidade da atenção oferecida.

\section{Hipervisibilidades}

As ações de saúde realizadas pelo CnaR estão norteadas, sobretudo, para o estabelecimento de vínculos com as pessoas em situação de rua, usando-se estratégias que compreendem a redução de danos e o olhar humanizador por meio da promoção, prevenção e cuidados primários em saúde fora dos espaços institucionais. Nesse sentido, as práticas de atenção voltadas para a população em situação de rua estão orientadas para proporcionar um acolhimento que respeite as escolhas e a autonomia dos indivíduos, livre de julgamentos, e que estimule a participação de cada indivíduo no cuidado 5 , reafirmando seus direitos de cidadãos brasileiros.

Basicamente, a demanda de procedimentos de saúde na rua consiste em avaliação clínica, aplicação de teste de diabete, curativos, aferição de pressão arterial, distribuição de água mineral e outros insumos, na orientação sobre cuidados em saúde e sobre os serviços assistenciais da Prefeitura, além, é claro, da escuta qualificada. Percorrer o centro da cidade e adentrar territórios considerados "perigosos" e "sujos" traz à tona que a rua não é só um lugar de passagem e circulação, é também local de permanência e de moradia, de repressão e de resistência 9 .

É indispensável lançar um olhar pormenorizado sobre essas questões e assumir a necessidade do enfrentamento da discriminação socioinstitucional à população em situação de rua, sobretudo para a melhoria do acolhimento nos serviços de saúde. A restrição de acesso imposta pelos próprios regulamentos das instituições públicas aos atores sociais marginalizados denuncia a necessidade de criação e efetivação de políticas e práticas institucionais sensíveis à pluralidade e pautadas na justiça e no reconhecimento social 16 . 
É pertinente então indagar como serviços e profissionais de saúde podem atender a uma crescente e complexa demanda de sujeitos em condições de rua em suas singularidades e necessidades? Como desenvolver outras possibilidades na organização do trabalho em saúde para superar a exclusão institucional da população em situação de rua? Carneiro \& Silveira 33 (p. 832) afirmam que é preciso problematizar a noção de exclusão social para assim "compreender $e$ dar respostas aos problemas de saúde dos vários grupos populacionais inseridos desigualmente na estrutura social (...) buscando entendimento para que se possa formular novos modos de organização das práticas que resultem em eficácia social".

Diante desse quadro, é preciso conferir hipervisibilidade à população em situação de rua, que sobrevive em condições de "não existência”. Em outras palavras, é preciso desenvolver um acolhimento que potencialize o encontro e promova uma relação de troca entre usuário e profissional de saúde. Segundo a Política Nacional de Humanização, antes de ser uma postura ética, o acolhimento é uma diretriz voltada para dar respostas às necessidades dos usuários dos serviços de saúde por meio do reconhecimento do paciente como protagonista do processo saúde/doença ${ }^{34}$.

Para isso, podemos tomar o acolhimento como uma ação de interculturalidade na saúde, que contribui, em grande escala, para (re)pensar o tipo de atendimento prestado a diferentes e distintos segmentos populacionais, sobretudo aqueles que são socialmente desfavorecidos 16 . Trata-se de assumir o acolhimento como encontro de aprendizagem mútua, que transcende o que está estabelecido pelas tecnociências da saúde e vai além do caráter normativo dos protocolos de atendimento, percorrendo um trajeto que viabilize os valores associados à felicidade humana por meio dos significados e sentidos não apenas à saúde, mas ao próprio projeto de vida dos indivíduos 14 .

Em reflexão socioantropológica, podemos colocar essa questão em termos de "interculturalidade", conceito que sumariamente denota qualquer relação entre pessoas e grupos sociais de diferentes culturas, bem como as atitudes de pessoas e grupos de uma cultura em relação a outra 19. De acordo com Carrons 19, no campo da saúde esse enfoque é fundamental, sobretudo quando as consequências da interlocução entre pessoas de origens culturais distintas podem afetar a qualidade de vida e assim garantir condições de sobrevivência aos indivíduos que estão sob cuidados médicos.

Nessa perspectiva descolonizadora, acolher remete a um cuidado emancipador 14 que está fundamentado no reconhecimento da diversidade e autonomia dos sujeitos, que permite, assim, a transformação do sujeito paciente-passivo em agente-participativo do seu processo de saúde, doença e cuidado. Tal condição ainda possibilita e potencializa a aproximação e interlocução entre profissionais de saúde e usuários, reconfigurando relações médico-centradas em relações que preservam (e até resgatam) as histórias de vida e os saberes dos indivíduos em tratamento.

Diante desse encontro, as "estratégias de aproximação”, que a equipe do CnaR lança mão, favorecem a criação de vínculos de respeito e confiança. Dessa maneira, ao mesmo tempo em que os profissionais do CnaR distribuem copos de água mineral para a população em situação de rua, conversas são desenvolvidas sobre a qual rede social os moradores de rua pertencem, suas preferências e suas formas de sociabilidade. É nessa interação que se estabelecem relações de empatia e se elege qual membro da equipe será referência de cada usuário do serviço.

\section{Considerações finais}

O CnaR foi criado como uma proposta de cuidado que amplia o acesso e a qualidade da atenção integral a sujeitos marginalizados. Orientado para buscar alternativas para as diversas problemáticas vivenciadas no contexto da rua, o CnaR oferece não apenas atenção em saúde, mas também garantia de direitos. Por exemplo, a possibilidade de confeccionar o Cartão SUS, que é um dos recursos que viabiliza não apenas o atendimento em serviços de saúde, mas o seu reconhecimento como cidadão em um ambiente onde geralmente são negados os seus direitos.

Usar a escuta como instrumento político ${ }^{9}$ - reconhecendo e valorizando a autonomia e a diversidade de saberes e narrativas dos sujeitos - nas ações em saúde contribui para uma verdadeira interação no encontro entre trabalhadores da saúde e pessoas em situação de rua, ao invés de focar apenas na intervenção terapêutica para a melhora clínica. Ao se buscar estratégias de hipervisibilização das possibilidades de cuidado emancipador para esse grupo, percebem-se situações adversas impostas pela vida na rua e as suas necessidades coletivas e individuais. A capacidade de colocar-se diante do "morador de rua" requer, assim, o rompimento com o "saber-poder colonizador" da lógica biomédica, que acaba por reduzir ou anular as singularidades e subjetividades dos indivíduos 16 .

Cada campo de atuação do CnaR apresenta particularidades e problemáticas diferentes, exigindo um tipo de observação específica para 
identificar como os indivíduos se associam, onde cada grupo se estabelece, como se organizam e como buscam recursos básicos para sobreviver na rua. Compreender o espaço da rua não como um lugar de passagem, visualizando-o como território e como lugar de permanências e ausências, com suas regras e conflitos, permite pensar a rua, se não como uma instituição, ao menos como uma organização social estruturante construída à margem da estrutura social dominante.

O cuidado da população em situação de rua instiga a refletir sobre os vários processos que estão inerentes ao movimento de visibilidade, invisibilidade e hipervisibilidade. A interculturalidade como ação política pode ser associada à estratégia de acolhimento e cuidado, podendo contribuir para uma mudança estrutural que vise a superar o distanciamento imposto pela discriminação social, que impede, entre outras coisas, a acessibilidade da população em situação de rua aos serviços do SUS.

A construção de um processo de trabalho em saúde pautado na abertura para o outro pos- sibilita uma legítima e necessária interlocução entre os profissionais de saúde e as pessoas em situação de rua. O encontro acolhedor pode ser colocado como produtor potencial de saúde, no qual a construção de vínculos de confiança é fundamental. A observação das características particulares de cada campo de atuação é primordial para criar linhas de cuidado que deem conta das situações demandadas por "viver na rua” e garantam autonomia por intermédio do potencial descolonizador do cuidado.

O desafio que segue, portanto, é criar possibilidades para que esse grupo social rompa com a invisibilidade e participe do projeto nação brasileira 16. Sendo assim, é importante que os profissionais de saúde acolham o morador de rua como sujeito de direitos e como um indivíduo portador de uma trajetória de vida singular. Conhecer os territórios, as dinâmicas de vidas e as suas práticas de transgressão é parte de um processo de abertura para o "outro" e o caminho para a desconstrução do olhar estigmatizante e colonizador.

\section{Resumen}

En este artículo se reflexiona sobre la producción de la atención a las personas sin hogar desde una perspectiva socio-antropológica, a partir de la observación participante realizada con un equipo de consulta médica en la calle. Se observó que esta población históricamente vista como marginal, apenas es capaz de acceder a los servicios de salud, por lo que es invisible para el propio Sistema Único de Salud de Brasil. Pese a que la Política Nacional de Población para Personas sin Hogar, garantiza el acceso a la atención médica, sin embargo, no siempre está garantizada, ya que los servicios y los profesionales de la salud tienen poca experiencia para acoger a las personas sin hogar. Incluso se llegó a la conclusión de que hay que hipervisibilizar las líneas necesarias de atención a situaciones que impone la vida en la calle y establecer lazos de corte deconstructivo entre el ámbito terapéutico y el de estigmatización.

\section{Colaboradores}

J. A. S. Hallais realizou o trabalho de campo, análise de dados e redigiu o artigo. N. F. Barros colaborou na análise de dados, concepção, redação e revisão do artigo.

\section{Agradecimentos}

À Fundação Desenvolvimento Administrativo (FUNDAP) pelo financiamento da bolsa no Programa de Aprimoramento Profissional, no curso Ciências Sociais em Saúde, realizado na Universidade Estadual de Campinas (Unicamp) em 2013. À equipe do Consultório na Rua de Campinas (2013): Alcyone, Felipe, Suzy, Tina, Magna, Carla, Carol, Thiago, Regis, Fabi, Lívia, Bárbara e Henrique pela acolhida e aprendizado em campo. 


\section{Referências}

1. Brasil. Decreto Presidencial no 7.053, de 23 de dezembro de 2009: institui a Política Nacional para a População em Situação de Rua e seu Comitê Intersetorial de Acompanhamento e Monitoramento, e dá outras providências. Diário Oficial da União 2009; 24 dez.

2. Brasil. Portaria no 122 , de 25 de janeiro de 2012 : define as diretrizes de organização e funcionamento das equipes de Consultório na Rua. Diário Oficial da União 2012; 26 jan.

3. Ministério da Saúde. Política Nacional de Atenção Básica. Brasília: Ministério da Saúde; 2012.

4. Ministério da Saúde. Manual sobre o cuidado à saúde junto à população em situação de rua. Brasília: Ministério da Saúde; 2012.

5. Jorge JS, Corradi-Webster CM. Consultório de rua: contribuições e desafios de uma prática em construção. Saúde \& Transformação Social 2012; 3:39-48.

6. Silva FP, Frazão IS, Linhares FMP. Práticas de saúde das equipes dos Consultórios de Rua. Cad Saúde Pública 2014; 30:805-14.

7. Barros NF. O ensino das ciências sociais em saúde: entre o aplicado e o teórico. Ciênc Saúde Coletiva 2014; 19:1053-63.

8. Durham ER. Bronislaw Malinowski: antropologia. São Paulo: Editora Ática; 1986.

9. Tuhiwai SL. Decolonizing methodologies: research and indigenous peoples. Dunedin: Zed Books; 2006.

10. Pereira PPG. De corpos e travessias: uma antropologia de corpos e afetos. São Paulo: Annablume; 2014. (Coleção Queer).

11. Moreira C. Tales of conde: autoethnography and the body politics of performative writing. Cultural Studies Critical Methodologies 2011; 11:586-95.

12. Magnani JGC. De perto e de dentro: notas para uma etnografia urbana. Rev Bras Ciênc Soc 2002; 17:11-29.

13. Ayres JRCM. O cuidado, os modos de ser (do) humano e as práticas em saúde. Saúde Soc 2004; 13:16-29.

14. Barros NF. As práticas integrativas e complementares como cuidado emancipador. https://cursos. atencaobasica.org.br/conteudo/8900 (acessado em 08/Mai/2015)

15. Starfield B. Atenção primária: equilíbrio entre necessidades de saúde, serviços e tecnologia. Brasília: Organização das Nações Unidas para a Educação, a Ciência e a Cultura, Ministério da Saúde; 2002.

16. Jardim DF, López LC, organizadores. Políticas da diversidade - (in)visibilidades, pluralidade e cidadania em uma perspectiva antropológica. Porto Alegre: Editora UFRGS; 2013.

17. Barros NF. Políticas da diversidade. Ciênc Saúde Coletiva 2014; 19:4923-4.

18. Garcia MTC. Salud e interculturalidad: reflexiones en torno a un caso. In: Juarez GF, editor. Salud e interculturalidad en América Latina: antropología de la salud y crítica intercultural. Quito: Abya-Yala; 2006. p. 109-14.

19. Corrons XA. Interculturalidad y salud. In: Juarez GF, editor. Salud e interculturalidad en América Latina: perspectivas antropológicas. Quito: AbyaYala; 2004. p. 65-74.
20. Siegel P, Barros NF. Análise sociológica das políticas de saúde. Ciênc Saúde Coletiva 2012; 17:1375-6.

21. Ministério da Saúde. HumanizaSUS: documento base para gestores e trabalhadores do SUS. 4a Ed. Brasília: Ministério da Saúde; 2008.

22. Secretaria de Cidadania, Assistência e Inclusão Social, Prefeitura Municipal de Campinas. Plano de Assistência Social do Município de Campinas (PMAS) - 2014/2017. Campinas: Prefeitura Municipal de Campinas; 2014.

23. Valencio NFLS, Pavan BJC, Siena M, Marchezini V. Pessoas em situação de rua no Brasil: estigmatização, desfiliação e desterritorialização. Revista Brasileira de Sociologia da Emoção 2008; 7:556-605.

24. Foucault M. Microfísica do poder. 13ạ Ed. Rio de Janeiro: Edições Graal; 1998.

25. Bourdieu P. Efeitos do lugar. In: Bourdieu P, organizador. A miséria do mundo. Petrópolis: Editora Vozes; 1997. p. 159-66.

26. Frugoli Jr. H, Sklair J. O bairro da Luz em São Paulo: questões antropológicas sobre o fenômeno da "gentrification". Cuadernos de Antropología Social 2009; (30):119-36.

27. Rui TC. Corpos abjetos: etnografia em cenários de uso e comércio de crack [Tese de Doutorado] Campinas: Programa de Pós-graduação em Antropologia Social, Universidade Estadual de Campinas; 2012.

28. Brasil. Portaria no 940 , de 28 de abril de 2011. Regulamenta o Sistema Cartão Nacional de Saúde (Sistema Cartão). Diário Oficial da União 2011; 2 mai.

29. Varanda W, Adorno RCF. Descartáveis urbanos: discutindo a complexidade da população de rua e o desafio para políticas de saúde. Saúde Soc 2004; 13:56-69.

30. Carneiro Jr. N, Nogueira EA, Lanferini GM, Ali DA, Martinelli M. Serviços de saúde e população de rua: contribuição para um debate. Saúde Soc 1998; 7:47-62.

31. Ministério da Saúde. Nota Técnica Conjunta/2012: adequação dos Consultórios de Rua e Implantação de Novas Equipes de Consultório na Rua, frente às diretrizes de funcionamento das equipes de Consultório na Rua, estabelecidas pelas portarias 122 e 123 de 25 de janeiro de 2012. Brasília: Ministério da Saúde; 2012.

32. Ministério da Saúde. Políticas de promoção da equidade em saúde. Brasília: Ministério da Saúde; 2013.

33. Carneiro Jr. N, Silveira C. Organização das práticas de atenção primária em saúde no contexto dos processos de exclusão/inclusão social. Cad Saúde Pública 2003; 19:1827-35.

34. Ministério da Saúde. Acolhimento nas práticas de produção de saúde. 2a Ed. Brasília: Ministério da Saúde; 2010.

Recebido em 19/Set/2014

Versão final reapresentada em 04/Fev/2015

Aprovado em 05/Fev/2015 